University of Nebraska - Lincoln

DigitalCommons@University of Nebraska - Lincoln

March 2002

\title{
Anisotropy and Magnetic Field Effects on the Entanglement of a Two Qubit Heisenberg $X Y$ Chain
}

\author{
G. Lagmago Kamta \\ University of Nebraska - Lincoln \\ Anthony F. Starace \\ University of Nebraska-Lincoln, astarace1@unl.edu
}

Follow this and additional works at: https://digitalcommons.unl.edu/physicsstarace

Part of the Physics Commons

Lagmago Kamta, G. and Starace, Anthony F., "Anisotropy and Magnetic Field Effects on the Entanglement of a Two Qubit Heisenberg XY Chain " (2002). Anthony F. Starace Publications. 83.

https://digitalcommons.unl.edu/physicsstarace/83

This Article is brought to you for free and open access by the Research Papers in Physics and Astronomy at DigitalCommons@University of Nebraska - Lincoln. It has been accepted for inclusion in Anthony F. Starace Publications by an authorized administrator of DigitalCommons@University of Nebraska - Lincoln. 


\title{
Anisotropy and Magnetic Field Effects on the Entanglement of a Two Qubit Heisenberg $X Y$ Chain
}

\author{
G. Lagmago Kamta and Anthony F. Starace \\ Department of Physics and Astronomy, The University of Nebraska, 116 Brace Laboratory, Lincoln, Nebraska 68588-0111
}

(Received 28 September 2001; published 26 February 2002)

\begin{abstract}
We investigate the entanglement of a two-qubit anisotropic Heisenberg $X Y$ chain in thermal equilibrium at temperature $T$ in the presence of an external magnetic field $B$ along the $z$ axis. By means of the combined influences of anisotropic interactions and a magnetic field $B$, one is able to produce entanglement for any finite $T$, by adjusting the magnetic field strength. This contrasts with the isotropic interaction or the $B=0$ cases, for which there is no entanglement above a critical temperature $T_{c}$ that is independent of the external $B$ field.
\end{abstract}

\section{DOI: 10.1103/PhysRevLett.88.107901}

Entanglement is a nonlocal correlation between quantum systems that does not exist classically. Entangled pairs of quantum systems remain strongly correlated even if they are well separated spatially; observing the state of one fixes with certainty the state of the other. In recent years, quantum entanglement has become recognized as crucial in various fields of quantum information, such as quantum cryptography [1], teleportation [2], and computation [3]. Potential applications of entanglement in these fields have stimulated research on ways to quantify and control it. Considerable attention has been devoted to interacting Heisenberg spin systems, which may be used for gate operations in solid state quantum computation processors [4-6]. Entanglement in isotropic Heisenberg spin chains has been studied both in the absence [7-9] and in the presence $[8,9]$ of an external magnetic field $B$ (along the $z$ axis). The magnetic field provides an additional parameter (besides the temperature $T$ ) for controlling entanglement. The concurrence (a measure of entanglement; see below) of a two-qubit isotropic Heisenberg system decreases with increasing $T$ and vanishes beyond a critical value $T=T_{c}$ $[8,9]$, which is independent of $B$. Also, the concurrence of a two-qubit isotropic Heisenberg chain decreases monotonically with increasing $B$ for any finite $T$ and vanishes exponentially with increasing $B[8,9]$. The corresponding anisotropic case has been investigated, but only for $B=0$ [9]; increasing the anisotropy in this case is also found to reduce monotonically the system's concurrence for any finite $T$ and to decrease the critical temperature $T_{c}$ beyond which the concurrence vanishes. In this work, we investigate the influence of anisotropy on the entanglement of a two-qubit Heisenberg system in the presence of an external $B$ field at thermal equilibrium. We demonstrate that the anisotropy and the magnetic field strength may together be used to control the extent of entanglement and, in particular, to produce entanglement at any finite $T$.

The Heisenberg Hamiltonian for a chain of $N$ spin $\frac{1}{2}$ systems having nearest-neighbor interactions is [10]

$$
H=\sum_{n=1}^{N}\left(J_{x} S_{n}^{x} S_{n+1}^{x}+J_{y} S_{n}^{y} S_{n+1}^{y}+J_{z} S_{n}^{z} S_{n+1}^{z}\right),
$$

PACS numbers: 03.65.Ud, 03.67.-a, 05.50.+q, 75.10.Jm

where $S_{n}^{\alpha}=\frac{1}{2} \sigma_{n}^{\alpha}(\alpha=x, y, z)$ denotes the local spin $\frac{1}{2}$ operator at site $n, \sigma_{n}^{\alpha}$ are the Pauli matrices at site $n$, and the periodic boundary condition $S_{N+1}=S_{1}$ applies. The $J_{\alpha}$ 's are real coupling constants for the spin interaction. For arbitrary $J_{\alpha}$ 's, the Heisenberg chain is often called the $X Y Z$ model, and the special cases $J_{x}=J_{y} \neq J_{z}$ (partial anisotropy) and $J_{x}=J_{y}=J_{z}$ (isotropy) are termed the $X X Z$ and $X X X$ models, respectively. The chain is said to be antiferromagnetic for $J_{\alpha}>0$ and ferromagnetic for $J_{\alpha}<0$. The Heisenberg interaction has been studied extensively in statistical physics (see, e.g., [11] and references therein) and mathematical physics [12], largely because of applications in solid state physics. Interest in this system has been revived recently by several proposals for realizing solid state quantum computation processors using localized electron spins as qubits, in which basic gate operations involve various forms of the Heisenberg Hamiltonian [4-6]. The $X Y\left(J_{z}=0\right)$ and the Heisenberg-Ising $\left(J_{y}=J_{z}=0\right)$ interactions are analyzed in the context of quantum gate operations in Refs. [5,6].

Consider now the Hamiltonian $H$ for the anisotropic two-qubit Heisenberg $X Y$ chain in an external magnetic field $B$ (along the $z$ axis) in terms of the raising and lowering operators $S^{ \pm}=S^{x} \pm i S^{y}$,

$$
\begin{aligned}
H= & B\left(S_{1}^{z}+S_{2}^{z}\right)+J\left(S_{1}^{+} S_{2}^{-}+S_{1}^{-} S_{2}^{+}\right) \\
& +J \gamma\left(S_{1}^{+} S_{2}^{+}+S_{1}^{-} S_{2}^{-}\right),
\end{aligned}
$$

where $J=\left(J_{x}+J_{y}\right) / 2$ and $\gamma=\left(J_{x}-J_{y}\right) /\left(J_{x}+J_{y}\right)$. The parameter $\gamma(-1 \leq \gamma \leq 1)$ measures the anisotropy of the system and equals 0 for the isotropic $X Y$ model and \pm 1 for the Ising model. The eigenvalues and eigenvectors of $H$ are given by $H\left|\Psi^{ \pm}\right\rangle= \pm J\left|\Psi^{ \pm}\right\rangle$and $H\left|\Sigma^{ \pm}\right\rangle= \pm \eta\left|\Sigma^{ \pm}\right\rangle, \quad$ where $\quad\left|\Psi^{ \pm}\right\rangle=\frac{1}{\sqrt{2}}(|01\rangle \pm|10\rangle)$ and $\left|\Sigma^{ \pm}\right\rangle=N^{ \pm}\left(|00\rangle \pm \frac{J \gamma}{\eta \overline{+B}}|11\rangle\right), \quad \eta=\sqrt{B^{2}+(J \gamma)^{2}}$, and the normalization constants are $N^{ \pm}=(\eta \mp B) /$ $\sqrt{(J \gamma)^{2}+(\eta \bar{\mp} B)^{2}}$. When $\gamma \neq 0$, the eigenvectors represent entangled states; also, when $B=0,\left|\Sigma^{ \pm}\right\rangle=$ $\left|\Phi^{ \pm}\right\rangle \equiv \frac{1}{\sqrt{2}}(|00\rangle \pm|11\rangle)$, so that $\left|\Psi^{ \pm}\right\rangle$and $\left|\Sigma^{ \pm}\right\rangle$are the four maximally entangled Bell states. 
Many measures of entanglement have been introduced and analyzed [13-17], but the one most relevant to this work is the "entanglement of formation" $E$ [13]. For a system in equilibrium at temperature $T$, the density operator is $\rho=Z^{-1} \exp \left(-H / k_{B} T\right)$, where $Z=\operatorname{tr}\left\{\exp \left(-H / k_{B} T\right)\right\}$ is the partition function and $k_{B}$ is Boltzmann's constant. For a pair of qubits, 1 and $2, E=h\left(\frac{1}{2}+\frac{1}{2} \sqrt{1-C^{2}}\right)$, where $h$ is the binary entropy function $h(x)=-x \log _{2} x-(1-$ $x) \log _{2}(1-x) . \quad C$ denotes the concurrence, defined as $[13,14]$

$$
C=\max \left\{\lambda_{1}-\lambda_{2}-\lambda_{3}-\lambda_{4}, 0\right\},
$$

where the $\lambda_{i}(i=1,2,3,4)$ are the square roots of the eigenvalues in decreasing order of magnitude of the "spin-flipped" density matrix operator $R=$ $\rho\left(\sigma^{y} \otimes \sigma^{y}\right) \rho^{*}\left(\sigma^{y} \otimes \sigma^{y}\right)$, where the asterisk indicates complex conjugation. Like $E$, the concurrence $C$ ranges from 0 (no entanglement) to 1 (maximum entanglement) and is a monotonically increasing function of $E$, so that $C$ is itself a measure of the entanglement [14]. As $\rho(T)$ describes a thermal state, such entanglement is referred to as thermal entanglement [8].

Using the Hamiltonian (2) one obtains the four eigenvalues of $R,\left(\lambda_{i}\right)^{2}$, as

$$
\begin{gathered}
\left(\lambda_{1,2}\right)^{2}=Z^{-2} \exp \left( \pm 2 J / k_{B} T\right), \\
\left(\lambda_{3,4}\right)^{2}=\frac{1}{Z^{2}}\left\{1+\frac{2 b^{2}}{\eta^{2}} \sinh ^{2}\left(\frac{\eta}{k_{B} T}\right)\right. \\
\left. \pm \frac{2 b}{\eta} \sinh \left(\frac{\eta}{k_{B} T}\right) \sqrt{1+\frac{b^{2}}{\eta^{2}} \sinh ^{2}\left(\frac{\eta}{k_{B} T}\right)}\right\}
\end{gathered}
$$

where $b=J \gamma, \quad Z=2\left[\cosh \left(\eta / k_{B} T\right)+\cosh \left(J / k_{B} T\right)\right]$, and $\lambda_{1,3}$ take the "+" sign in Eqs. (4) and (5), while $\lambda_{2,4}$ take the "-" sign. The $\lambda_{i}$ are in arbitrary order. Since their relative magnitudes depend on the parameters involved, they cannot be ordered by magnitude unless the parameter values are known. This prevents one from writing a more specific analytical expression for $C$ than (3). For particular parameters, $C$ can be evaluated numerically or even analytically; e.g., for $B=0$ and $\gamma=0$ the above expressions for $\lambda_{i}$ yield the concurrences obtained in Ref. [9]. Note that $C$ vanishes in the limits $B \rightarrow \infty$ or $T \rightarrow \infty$. The concurrence derived from Eqs. (3)-(5) is invariant under the substitutions $J \rightarrow-J$, which indicates that the entanglement is the same for the antiferromagnetic and ferromagnetic cases. The concurrence is also the same for $\gamma$ and $-\gamma$. Therefore, we restrict our considerations to $J>0$ and $0 \leq \gamma \leq 1$.

For anisotropy parameters $\gamma=0,0.3,0.6$, and 1, plots of the concurrence $C$ as a function of both $k_{B} T$ and $B$ are given in Figs. 1 and 2. Note the qualitative similarity of the present results for $C$ in the isotropic case [Fig. 1(a)] to the entanglement of formation $E$ for the isotropic $X Y Z$ model presented in Fig. 1 of Ref. [8]. The results for $\gamma=0,0.3$, and 0.6 have the similar feature that $C$ is maximal when $T=0$ and $B=0$. This is because at $T=$ 0 the system is in its ground state, which is the maximally entangled Bell state $\left|\Psi^{-}\right\rangle$.

Consider first the isotropic case [Fig. 1(a)] at $T=0$. With increasing $B$, the concurrence is constant and maximal, but it drops suddenly and vanishes as $B$ crosses the critical value $B=J$. Indeed, for $B>J$, the ground state for this case is the unentangled state $|00\rangle$. At the critical point $\left(T=0, B=B_{c}=J\right)$, the entanglement becomes a nonanalytic function of $B$ and a quantum phase transition [18] occurs. Such transitions have been found also in the isotropic $X Y$ [9] and $X Y Z$ [8] models.

The anisotropic cases in Figs. 1(b) and 1(c) show novel features, which we consider first for $T=0$. As $B$ increases, $C$ is initially constant and equal to its maximum value. It then decreases suddenly as a critical field $B_{c}$ is reached, similarly to the isotropic case. However, instead of vanishing for $B>B_{c}$, the concurrence persists and, for sufficiently large $\gamma$, undergoes a revival (i.e., an increase in magnitude) before decreasing to zero. For $T=0$, Eqs. (3)-(5) allow $C$ to be written analytically as
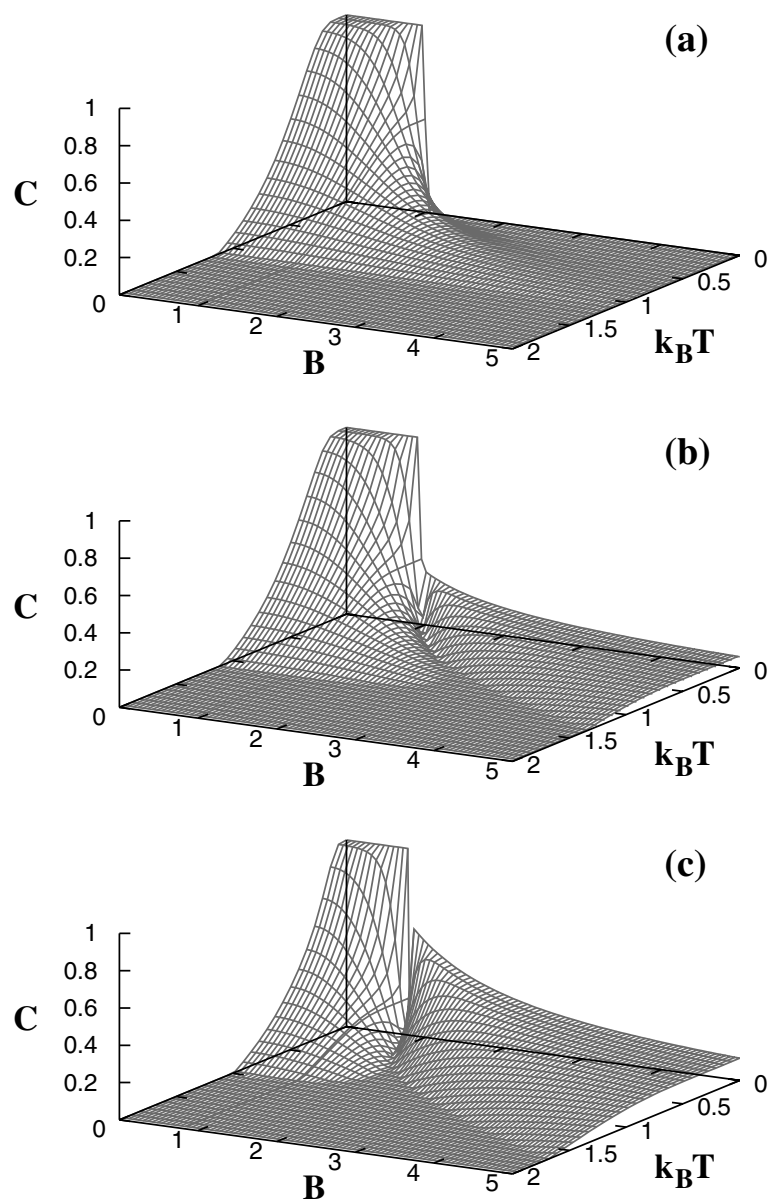

FIG. 1. Concurrence $C$ in the Heisenberg $X Y$ chain of two qubits plotted vs $k_{B} T$ (the Boltzmann constant times temperature) and $B$ (the external magnetic field), for various values of the anisotropy parameter: (a) $\gamma=0$, (b) $\gamma=0.3$, and (c) $\gamma=0.6$. The coupling constant is $J=1$. 


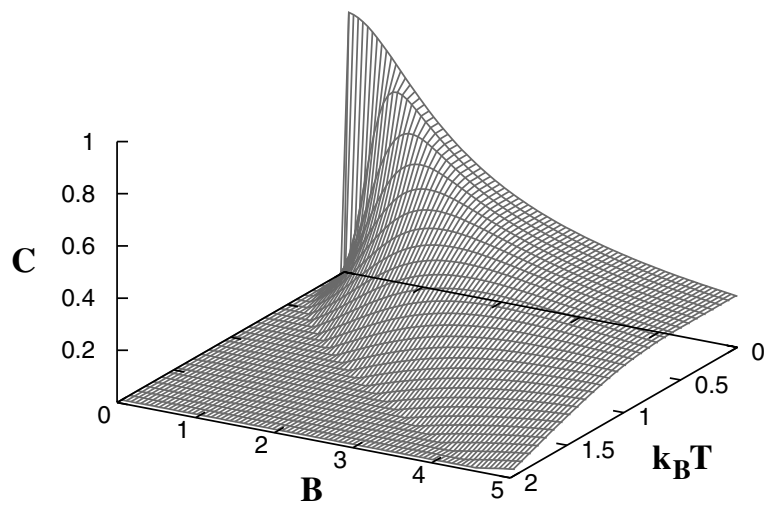

FIG. 2. Same as Fig. 1 for $\gamma=1.0$ (Ising model).

$$
C(T=0)= \begin{cases}1 & \text { for } \eta<J \\ (1-|\gamma|) / 2 & \text { for } \eta=J \\ |J \gamma| / \eta & \text { for } \eta>J\end{cases}
$$

It follows that the critical field $B_{c}$ at which $C$ becomes a nonanalytic function of $B$ is given by $\eta=J$ or $B_{c}=J \sqrt{1-\gamma^{2}}$. Therefore a quantum phase transition also occurs in the anisotropic $X Y$ chain at $T=0$ for $B=B_{c}$, but without the entanglement vanishing. Moreover, for $\gamma>\frac{1}{3}$ there occurs a revival of the entanglement for magnetic fields $B \gtrsim B_{c}$, as $C(B)$ becomes larger than its value at $B=B_{c}$ [cf. Fig. 1(c) for $\gamma=0.60]$. Figure 3 shows the concurrence at $T=0$ for $\gamma=0,0.33,0.60,0.80$, and 1.0. For $\gamma=0.33$, $C$ drops sharply to a finite value for $B=B_{c}$; for

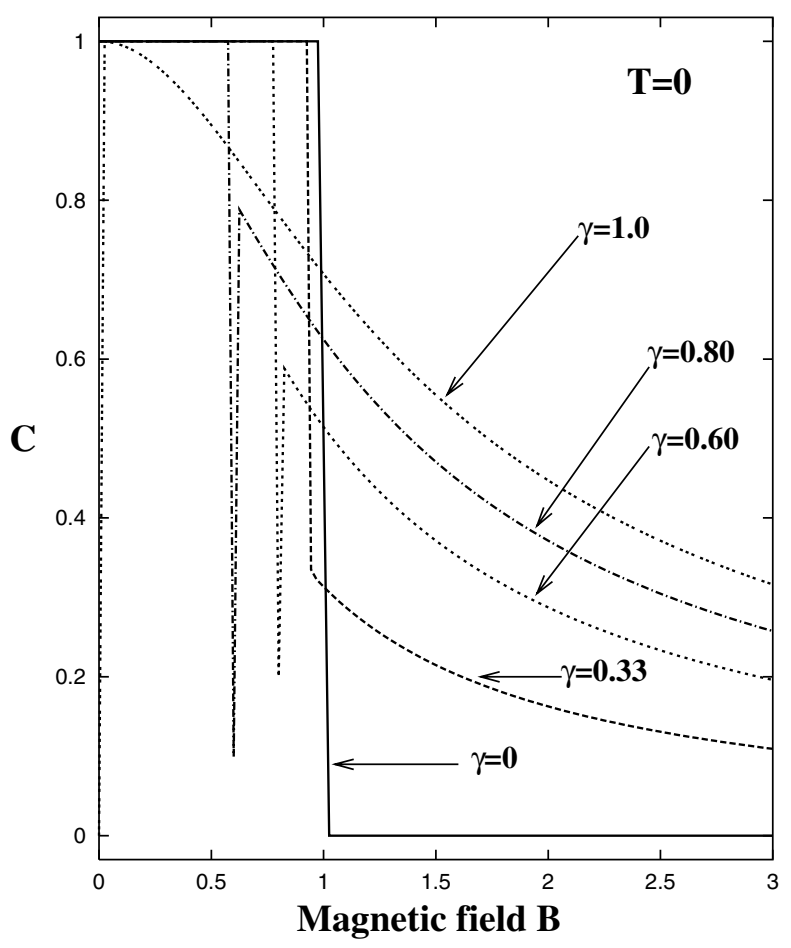

FIG. 3. Concurrence $C$ vs the magnetic field strength $B$ at zero temperature for various values of the anisotropy parameter $\gamma$, with $J=1$. Note that for $\gamma=1.0, C(B=0)=0$. Note also the revival in $C$ for values of $\gamma>0.33$ for $B \gtrsim B_{c}$, where $B_{c}=0.8,0.6,0$ for $\gamma=0.60,0.80,1.0$, respectively.
$B>B_{c}, C$ decreases monotonically. For $\gamma>1 / 3, C$ drops sharply at $B=B_{c}$, but for $B \gtrsim B_{c}, C$ exhibits a revival before decreasing to zero asymptotically (cf. Fig. 3 for $\gamma=0.60$ and $\gamma=0.80$ ).

The system's behavior at the quantum phase transition point $B=B_{c}$ may be determined from the density matrix at $T=0$, at which the system is in its ground state. When $\eta<J$ the ground state of the system is the maximally entangled state $\left|\Psi^{-}\right\rangle$, and when $\eta>J$, the ground state of the system is $\left|\Sigma^{-}\right\rangle$, which is also entangled. On the other hand, when $\eta=J,\left|\Psi^{-}\right\rangle$and $\left|\Sigma^{-}\right\rangle$have the same energies, equal to the lowest energy of the system. It follows that in general, the system at $T=0$ is a statistical mixture of $\left|\Psi^{-}\right\rangle$and $\left|\Sigma^{-}\right\rangle$and can be described using the mixed state density operator

$$
\rho=P_{\Psi}\left|\Psi^{-}\right\rangle\left\langle\Psi^{-}\left|+P_{\Sigma}\right| \Sigma^{-}\right\rangle\left\langle\Sigma^{-}\right|,
$$

where $P_{\Psi}$ and $P_{\Sigma}$ (with $P_{\Psi}+P_{\Sigma}=1$ ) are the probabilities of the two states. This density operator yields

$$
C(T=0)=\max \left\{0,\left|P_{\Psi}-P_{\Sigma}(|J \gamma| / \eta)\right|\right\} .
$$

For the $\eta<J$ and $\eta>J$ cases, respectively, which correspond to $\left(P_{\Psi}=1, P_{\Sigma}=0\right)$ and $\left(P_{\Psi}=0, P_{\Sigma}=1\right)$, Eq. (8) leads to $C=1$ and $C=|J \gamma| / \eta$. At the critical field $B=B_{c}$ (where $\eta=J$ ), Eq. (6) gives $C=(1-|\gamma|) / 2$. Comparing with Eq. (8) one sees that there is equality for $P_{\Psi}=P_{\Sigma}=1 / 2$. Thus the quantum phase transition occurs at $T=0$ when the ground state of the system is an equal mixture of $\left|\Psi^{-}\right\rangle$and $\left|\Sigma^{-}\right\rangle$and has a nonzero entanglement. As $B$ crosses $B_{c}$, the system switches isothermally from the statistically mixed ground state to the pure state $\left|\Sigma^{-}\right\rangle$. For $\gamma \leq \frac{1}{3}, C$ does not increase for $B \geq B_{c}$; for $\gamma>\frac{1}{3}$, however, $C$ exhibits a revival, i.e., it increases for $B \gtrsim B_{c}$, reaches a maximum, and then decreases. One sees from (6) that $C$ decreases as $\eta^{-1}$, which implies $C(B) \sim B^{-1}$ asymptotically.

In the Ising case $(\gamma=1)$, shown in Fig. 2 , there is no entanglement for $B=0$ [9], i.e., entanglement exists solely because of the magnetic field. In fact, one sees from Figs. 1 and 3, that, with increasing anisotropy parameter $\gamma$, the phase transition point at $T=0$ moves toward lower fields and that for $\gamma=1, B_{c}=0$. Therefore for $B=0$ and $T=0$, the Ising system is in a state whose entanglement is zero because it is an equal mixture of the two Bell states $\left|\Psi^{-}\right\rangle$and $\left|\Phi^{-}\right\rangle$. One may confirm this by showing that the density matrix, $\rho=\frac{1}{2}\left|\Psi^{-}\right\rangle\left\langle\Psi^{-}\left|+\frac{1}{2}\right| \Phi^{-}\right\rangle\left\langle\Phi^{-}\right|$, which describes this equal mixture, can be rewritten as $\rho=\frac{1}{2}|\psi(1)\rangle\langle\psi(1)| \otimes$ $|\phi(2)\rangle\left\langle\phi(2)\left|+\frac{1}{2}\right| \phi(1)\right\rangle\langle\phi(1)|\otimes| \psi(2)\rangle\langle\psi(2)|, \quad$ where $|\psi\rangle=\frac{1}{\sqrt{2}}(|0\rangle+|1\rangle), \quad|\phi\rangle=\frac{1}{\sqrt{2}}(|0\rangle-|1\rangle)$, and where the arguments (1) and (2) refer to the spins 1 and 2. The latter form of $\rho$ is separable, as it is expressed as a sum of products of reduced density matrices pertaining to each of the spins 1 and 2, weighted by their probability distributions. This separability of the density operator indicates that the mixture is in fact not entangled $[16,19]$. 
More generally, one may show similarly the unexpected result that any equal mixture of two maximally entangled Bell states is factorizable, i.e., not entangled.

Consider now the case $T>0$, for which no quantum phase transition occurs [18]. In this case, $C$ cannot be written analytically in as simple a form as Eq. (6); rather, one must obtain $C$ numerically using Eqs. (3)-(5). As $T$ increases for $B<B_{c}$ the entanglement decreases monotonically as the maximally entangled ground state $\left|\Psi^{-}\right\rangle$ becomes mixed with other states, and similarly for $B>$ $B_{c}$ as $\left|\Sigma^{-}\right\rangle$becomes mixed with other states. In every case (cf. Figs. 1 and 2) there exists for any $B$ a temperature above which the entanglement vanishes identically. This critical temperature $T_{c}$ is independent of $B$ in the isotropic case [Fig. 1(a)] and is given by $k_{B} T_{c}=1.1346 J$ [9]. In contrast to the isotropic case, $T_{c}$ in the anisotropic case is a function of $J, B$, and of $\gamma$. This is illustrated in Fig. 4, which is a contour plot extracted from Figs. 1 and 2 . It shows at what temperature $C$ vanishes as a function of the magnetic field $B$. On this plot, when there are two values of $T$ (for fixed $B$ ) for which $C$ vanishes, there is a revival of $C$ as a function of $T$, with $C$ vanishing above the highest value of $T$. Correspondingly, for fixed $T$, when there are two critical values of $B$ for which $C$ vanishes, $C$ is zero for $B$ between these two values; for $B$ above the

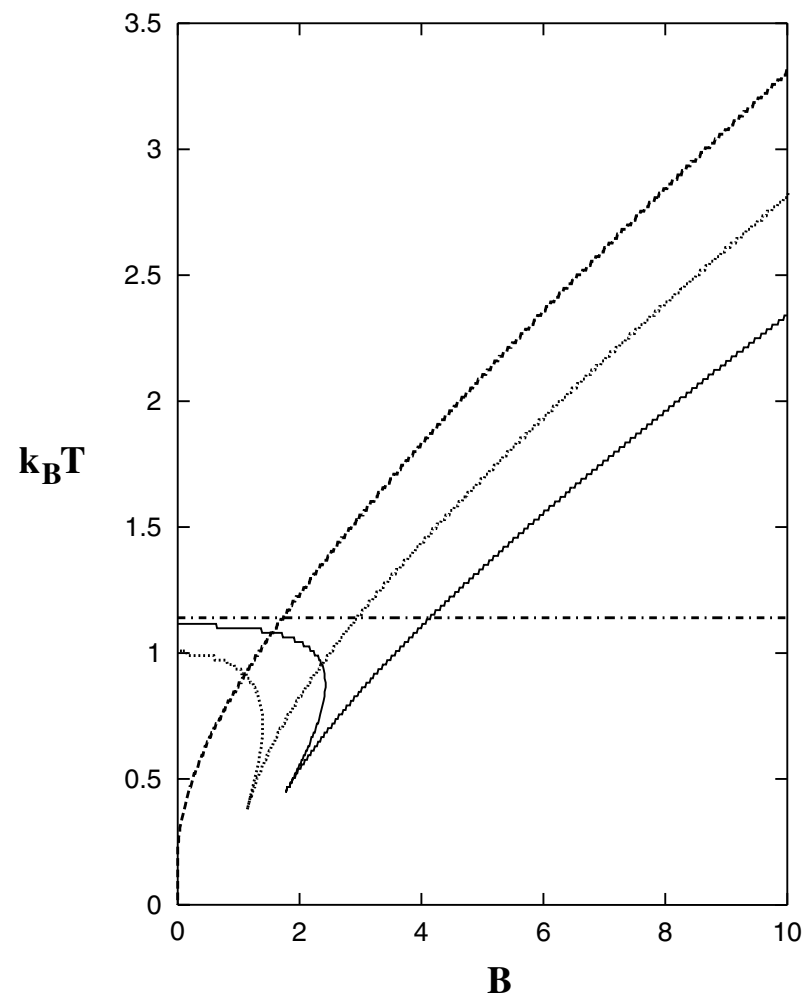

FIG. 4. Temperature at which the concurrence $C$ vanishes, plotted as a function of the magnetic field strength for various values of the anisotropy parameter: $\gamma=0$ (dot-dashed line), $\gamma=0.4$ (solid line), $\gamma=0.8$ (dotted line), and $\gamma=1$ (dashed line). In each case, $C=0$ in the region bounded by (and generally above) the relevant curve. Note that for any finite $T$, there is a $B$ for which $C>0$. second critical value, $C$ undergoes a revival and a smooth decay (as $B^{-1}$ for large $B$, in contrast to the exponential decrease with $B$ for $\gamma=0)$. For fixed $T>T_{c}(B=0)$, for which $C=0$ in the absence of a magnetic field, sufficiently large values of $B$ induce entanglement $(C>0)$, as shown in Figs. 1, 2, and 4 for $k_{B} T \gtrsim 1.2$. As shown in Fig. 4, for sufficiently large $B, T_{c}$ increases with both $\gamma$ and $B$. If fact, one may show analytically that for large $B$, the critical temperature $T_{c}$ at which $C$ vanishes is given by $k_{B} T_{c}=B / \log (4+2 B / b)$. These results show that the anisotropy permits one to obtain entangled qubits at higher $T$ and higher $B$ than is possible in the isotropic case. In the Ising case, where $B_{c}=0$, for $T<T_{c}$ the entanglement increases (from zero for $B=0$ ) up to a maximum and then decreases analytically to zero.

In conclusion, our study of the anisotropic two-qubit $X Y$ model in an external magnetic field $B$ reveals the strong combined influence of the anisotropy parameter $\gamma$ and the magnetic field on the entanglement. $\quad \gamma$ and $B$ produce entanglement in regions of $(B, T)$ parameter space where none exists in the isotropic case. Most importantly, they provide the means to control and produce entanglement in two spin systems for any finite $T$.

This work was supported by the Department of Energy, BES, Division of Chemical Sciences, under Grant No. DE-FG03-96ER14646, and by the Nebraska Research Initiative.

[1] A. K. Ekert, Phys. Rev. Lett. 67, 661 (1991).

[2] C. H. Bennett et al., Phys. Rev. Lett. 70, 1895 (1993).

[3] C. H. Bennett and D. P. DiVincenzo, Nature (London) 404, 247 (2000).

[4] D. Loss and D. P. DiVincenzo, Phys. Rev. A 57, 120 (1998); G. Burkard et al., Phys. Rev. B 59, 2070 (1999).

[5] A. Imamoḡlu et al., Phys. Rev. Lett. 83, 4204 (1999).

[6] R. Raussendorf and H. J. Briegel, Phys. Rev. Lett. 86, 5188 (2001).

[7] W. K. Wootters, quant-ph/0001114; K. M. O'Connor and W. K. Wootters, Phys. Rev. A 63, 052302 (2001).

[8] M. C. Arnesen et al., Phys. Rev. Lett. 87, 017901 (2001).

[9] X. Wang, Phys. Rev. A 64, 012313 (2001).

[10] V. E. Korepin, N. M. Bogoliubov, and A. G. Izergin, Quantum Inverse Scattering Method and Correlation Functions (Cambridge University Press, Cambridge, 1993), pp. 63-79.

[11] Z. N. C. Ha, Quantum Many-Body Systems in One Dimension (World Scientific, Singapore, 1996).

[12] E. Lieb et al., Ann. Phys. (N.Y.) 16, 407 (1961).

[13] C. H. Bennett et al., Phys. Rev. A 54, 3824 (1996).

[14] S. Hill and W. K. Wootters, Phys. Rev. Lett. 78, 5022 (1997); W. K. Wootters, Phys. Rev. Lett. 80, 2245 (1998).

[15] V. Vedral et al., Phys. Rev. Lett. 78, 2275 (1997).

[16] M. Horodecki et al., Phys. Rev. Lett. 80, 5239 (1998).

[17] E. M. Rains, Phys. Rev. A 60, 173 (1999); 60, 179 (1999).

[18] S. Sachdev, Quantum Phase Transitions (Cambridge University Press, Cambridge, 1999); Phys. World 12, 33 (April 1999).

[19] M. A. Nielsen and J. Kempe, Phys. Rev. Lett. 86, 5184 (2001). 